\title{
Miniaturization and embedding of soliton-based electro-optically addressable photonic arrays
}

\author{
Angelo D'Ercole, Elia Palange, and Eugenio DelRe \\ Dipartimento di Ingegneria Elettrica e dell'Informazione, \\ Università dell'Aquila, 67040 Monteluco di Roio-L'Aquila, Italy \\ Tel.: +390862 434364; Fax: +390862 434403; E-mail: edelre@ing.univaq.it \\ Alessandro Ciattoni and Bruno Crosignani \\ Dipartimento di Fisica, Universita' dell'Aquila, 67010 L'Aquila, Italy \\ and Istituto Nazionale Fisica della Materia - Consiglio Nazionale delle Ricerche, \\ Unita' di Roma "La Sapienza", 00185 Rome, Italy \\ Aharon J. Agranat \\ Applied Physics Department, Hebrew University of Jerusalem, Jerusalem 91904, \\ Israel
}

\begin{abstract}
Using top-electrodes, we demonstrate the soliton-based miniaturized integration of electro-optic devices in a photorefractive paraelectric bulk crystal. Self-trapping and beam manipulation though soliton electro-activation is achieved at quasi-digital voltages. (C) Optical Society of America 2006

OCIS codes: (230.2090) Electro-optical devices; (190.5330) Photorefractive nonlinear optics
\end{abstract}

Photorefractive spatial solitons [1] can be implemented to steer beams [2] and to achieve electro-optically activated beam manipulation functions, such as intensity modulation and two-channel routing, the devices themselves self-integrated in the bulk [3,4]. The optical circuitry is at once integrated in the bulk crystal volume, transparent at infrared wavelengths, and compatible with a fast response.

To date, no physical mechanism has been devised to allow for the possibility of imprinting a given steering pattern in the volume, formed through different localized soliton processes, and to subsequently address and electro-optically activate them through appropriate control signals, involving accessibly low switching voltages. We here demonstrate a viable avenue based on delivering the bias voltage through a single facet geometry, with all the electrodes deposited side-by-side, during the nonlinear propagation writing stage and in the subsequent routing stage [5]. Electro-statically, in this configuration, the values of external bias required to achieved $\mathrm{kV} / \mathrm{cm}$ fields can be considerably reduced by microscopically reducing the electrode distance. Furthermore, by tailoring the electrode geometry, we can achieve a quasi-arbitrary spatial pattern of the bias electric field, up to several hundred microns inside the sample.

We implement the geometry illustrated in Fig.(1a), which constitutes, in our scheme, the basic building block to the more complex and elaborate addressable photonic array $[6,7,8]$. We carry out experiments, in a $3^{(x)} \times 2.4^{(y)} \times 1^{(z)} \mathrm{mm}$ sized sample of $\mathrm{Cu}$ and $\mathrm{V}$ co-doped potassium-lithium-niobate-tantalate (KLTN) crystal, at a temperature $T=16^{\circ} \mathrm{C}, 4$ degrees above the ferro-paraelectric transition, where the index of refraction is $n \simeq 2.4$, the quadratic electro-optic coefficient $g \simeq 0.12 m^{4} C^{-2}$, and the relative dielectric constant $\epsilon_{r} \simeq 1.5 \cdot 10^{4}$. The top-sided electrodes were tailored on the $3^{(x)} \times 1^{(z)} \mathrm{mm}$ facet, with an intra-electrode 180 $\mu \mathrm{m}$ gap (see Fig.(1b)). The beam was a continuous-wave Argon ion $50 \mathrm{~mW} x$-polarized laser, operating at $\lambda=514 \mathrm{~nm}$ whose intensity distribution is approximated by a Gaussian of intensity Full-Width-at-HalfMaximum (FWHM) $\Delta x=\Delta y=7 \mu m$ and intensity ratio between the maximum beam intensity and the homogeneous background illumination $I_{\max } / I_{b}=5$.

Experimental results for a launch approximately $50 \mu \mathrm{m}$ from the top edge, are reported in Fig.(2). For a zero applied voltage, the beam diffracts to $15 \mu \mathrm{m}$ after the $1 \mathrm{~mm}$ propagation along the $z$ axis, corresponding to approximately 2 diffraction lengths (see Fig.(2b)). As predicted through numerical simulation, two-dimensional self-trapping is achieved for a $V_{\text {sol }} \simeq 40-50 \mathrm{~V}$, after a build-up time of approximately $\tau_{s} \simeq$ $120 \mathrm{~s}$, for a $1 \mu \mathrm{W}$ launch.

Similar self-trapping phenomenology was observed from 50-300 $\mu \mathrm{m}$ from the top edge, with voltages ranging from 40-160 V, leading to a screening-like soliton supporting region inside the crystal of $200^{(x)} \times 250^{(y)}$ $\mu \mathrm{m}^{2}$, schematically illustrated in Fig.(1b-c). The measured values of $V_{\text {sol }}$ at different distances from the edge are shown in Fig.(2d). Beams launched less than $50 \mu \mathrm{m}$ from the top edge experienced distortive edge- 
effects, whereas trapping beyond the $300 \mu \mathrm{m}$ limit required values of applied voltage leading to the dielectric breakdown of the air. In the horizontal dimension, the region is determined by the emergence of $y$-oriented components, which considerably weaken self-action.

By impinging on the pattern with a less intense $50 \mathrm{nW}$ read-out beam, which activates charge displacement only for times greater than $20 \tau_{s}$, we are able to achieve electro-optic $25 \mathrm{~dB}$ attenuation. This occurs because the soliton pattern, activated for $V_{a c t}=V_{\text {sol }}$, reverts to a defocusing structure for $V_{\text {act }}=0$ [3].

The next step was to implement our results to miniaturize electro-optic beam manipulation and switching, realizing a two-mode router using two solitons of opposite electro-optic response [3, 4], achieved within the same soliton-active region. The writing stage is accomplished in two steps: in the first, a single soliton is formed $90 \mu \mathrm{m}$ from the crystal edge, with an appropriate value of $V_{\text {sol }}$; subsequently, a second soliton is formed with an opposite biasing voltage $-V_{\text {sol }}$ at $110 \mu \mathrm{m}$ from the edge, such that they are vertically stacked. The first soliton is now activated and guides for a $V_{a c t}=V_{\text {sol }}$, the second being antiguiding, whereas for $V_{a c t}=-V_{\text {sol }}$, the roles are inverted, attaining the required routing, when the read beam is launched in between the two, at $100 \mu \mathrm{m}$ from the edge. Switching results and the relative predicted electro-optic patterns are shown in Fig.(3).

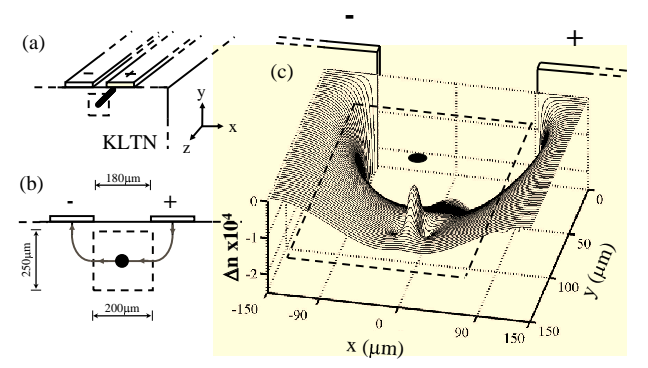

Fig. 1. Top-sided electrode geometry: schematic (a); detail of soliton-activated region (b); and numerical prediction of trapping pattern $\Delta n(x, y)$ (c).
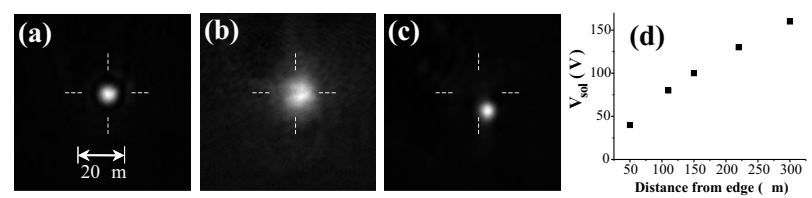

Fig. 2. Soliton formation: intensity distribution of the input $7 \mu \mathrm{m}$ FWHM beam (a); diffraction at output face (b); and self-trapped soliton for $V_{s o l}=40 \mathrm{~V}$ (c). Note the soliton slippage in the vertical direction due to the slight index gradient (see Fig.(1c)), combined to the standard self-bending process. Measured $V_{\text {sol }}$ versus the distance from the crystal edge is plotted in $(\mathrm{d})$.
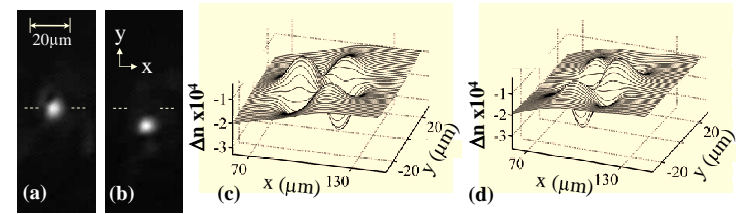

Fig. 3. Embedded routing: output intensity distribution for $V_{a c t}=80 \mathrm{~V}$ (a); for $V_{\text {act }}=-80 \mathrm{~V}$ (b); (c,d) the respective calculated index patterns $\Delta n(x, y)$.

\section{References}

1. M. Segev, B. Crosignani, A. Yariv, and B. Fischer, Phys.Rev.Lett 68, 923 (1992)

2. M.F. Shih, M. Segev, and G. Salamo, Opt.Lett. 21, 931 (1996)

3. E. DelRe, B. Crosignani, P. Di Porto, E. Palange, and A.J. Agranat, Opt.Lett. 27, 2188 (2002)

4. E. DelRe, E. Palange, and A.J. Agranat, J.Appl.Phys. 95, 3822 (2004)

5. A. DErcole, E. Palange, E. DelRe, A. Ciattoni, B. Crosignani, and A. J. Agranat, Appl.Phys.Lett. 85, 2679 (2004)

6. J.W. Fleischer, M. Segev, N.K. Efremidis, and D.N. Christodoulides, Nature 422, 147 (2003)

7. J. Petter, J. Schroder, D. Trager, and C. Denz, Opt.Lett. 28, 438 (2003)

8. P. Dittrich, G. Montemezzani, P. Bernasconi, and P. Gunter, Opt.Lett. 24, 1508 (1999) 